\title{
ESTUDIO DE LA VARIACIÓN DE LA GENITALIA MASCULINA DE LAS ESPECIES DE LA TRIBU PHANAEINI (SCARABAEIDAE: SCARABAEINAE) EN COLOMBIA*
}

\author{
Elsa H. Manjarres-H. ${ }^{1}$, Fredy Molano-R. ${ }^{2}$
}

\section{Resumen}

En este trabajo se examinó la variación morfológica de la genitalia masculina de la tribu Phanaeini, grupo de escarabajos coprófagos de importancia ecológica por el proceso de reciclaje de nutrientes que llevan a cabo. Se revisaron 122 ejemplares de 55 especies y 25 ejemplares de países como Costa Rica, Brasil, Perú, Bolivia y Venezuela. Se presenta la descripción de la variación del segmento genital, edeago, saco interno, esclerito basal, esclerito medial, esclerito placa y esclerito elongado de las especies de los seis géneros de la tribu Phanaeini presentes en Colombia. Los resultados de este trabajo muestran que la genitalia masculina de las especies de la tribu Phanaeini es heterogénea pero única en cada especie siendo de valor taxonómico; la diversificación en la morfología de la genitalia es bastante amplia, representada en la variación de las múltiples formas que adoptan principalmente los escleritos del saco interno.

Palabras clave: escarabajos coprófagos, escleritos, edeago, segmento genital, saco interno.

\section{STUDY OF THE VARIATION OF THE MALE GENITALIA SPECIES OF THE TRIBE PHANAEINI (SCARABAEIDAE: SCARABAEINAE) EN COLOMBIA}

\begin{abstract}
In this paper we examined the variation of male genitalia Phanaeini Tribe, a dung beetles group with ecological importance by nutrient recycling process carried out. We reviewed 122 specimens of 55 species, 25 specimens of countries such as Costa Rica, Brazil, Peru, Bolivia and Venezuela. We present the description of the variation of genital segment, aedeagus, internal sac, sclerite basal, sclerite medial, sclerite elongate and sclerite plate of the species of the six genera of Phanaeini tribe present in Colombian. The results of this work show that the male genitalia of species of the tribe Phanaeini are heterogeneous, but unique to each species being of taxonomic value; the diversification in morphology of the genitalia is sufficiently wide, variation represented in the multiple forms of the sclerites in the internal sac.
\end{abstract}

Key words: dung beetles, sclerites, aedeagus, genital segment, internal sac.

\footnotetext{
*FR: 6-X-2014 . FA: 1-III-2015.

${ }^{1}$ Bióloga, M. Sc. Laboratorio de Entomología, Museo de Historia Natural "Luis Gonzalo Andrade”, Universidad Pedagógica y Tecnológica de Colombia. Docente Departamento de Biología y Microbiología, Universidad de Boyacá. Tunja, Colombia. E-mail: ehmanjarresh@unal.edu.co

${ }^{2}$ Biólogo, M. Sc. Laboratorio de Entomología, Museo de Historia Natural "Luis Gonzalo Andrade”, Universidad Pedagógica y Tecnológica de Colombia. Docente asistente Universidad Pedagógica y Tecnológica de Colombia. Tunja, Colombia. E-mail: fredymol@gmail.com
} 


\section{INTRODUCCIÓN}

Los escarabeidos (Scarabaeidae) son una familia de coleópteros que comprende aproximadamente 30.000 especies en el mundo (HALFFTER, 1991), presentan una gran diversidad, tanto en modos de vida como en aspecto. Los escarabajos coprófagos (Scarabaeinae) por su parte son un gremio bien definido de esta familia y son muy importantes en el proceso de reciclaje de nutrientes en un ecosistema, además pueden verse afectados por las alteraciones medioambientales, lo que hace de este un taxón ideal para la investigación sobre la biodiversidad y conservación, siendo un grupo bioindicador de perturbaciones en diferentes hábitats (HALFFTER \& MATTHEWS, 1966; CAMBEFORT, 1991; HALFFTER \& FAVILA, 1993; SPECTOR, 2006).

Dentro de los Scarabaeidae, la tribu Phanaeini es la más conocida taxonómica y biogeográficamente de la región Neotropical (EDMONDS, 1972, 1994, 2000; ARNAUD, 2002; EDMONDS \& ZÍDEK, 2004; PHILIPS et al., 2004; PRICE, 2009), en Colombia se encuentran seis de los doce géneros de esta tribu (Oxysternon, Coprophanaeus, Phanaeus, Sulcophanaeus, Diabroctis, Gromphas) (MEDINA et al., 2001; NORIEGA et al., 2015). El primer acercamiento taxonómico de la tribu Phanaeini lo realizó Gregor d'Olsoufieff en 1924 presentando cinco claves para las especies de la tribu, además de proponer varias agrupaciones supraespecíficas; a partir de la monografía de d'Olsoufieff se realizaron diversos trabajos taxonómicos sobre el grupo dentro de los cuales están los de PESSÔA (1934), EDMONDS (1972), EDMONDS (1994), ARNAUD (2002), EDMONDS \& ZÍDEK (2010), entre otros.

Desde la perspectiva morfológica, los genitales masculinos constituyen unas de las estructuras más diversas en el reino animal existiendo un sinnúmero de diseños (CÓRDOBA-AGUILAR, 2000), por lo tanto la historia del uso de la estructura del aparato genital para diferenciar especies cuenta ya con más de un siglo de antigüedad. A lo largo de este tiempo, lo métodos de estudio han ido mejorando considerablemente (ANICHTCHENKO, 2004). Los caracteres del órgano genital masculino son ampliamente utilizados en la sistemática y taxonomía de todo el orden Coleoptera debido a la dificultad en la separación de especies por caracteres morfológicos, pues en algunos casos las especies son cripticas y con nichos ecológicos compartidos, es por esto que el uso de la morfología del órgano genital de los machos ha sido útil para separar y definir grupos naturales de especies (LÓPEZ-GUERRERO, 1999).

Sin embargo, a pesar de que la diversidad de la morfología genital es un tópico muy interesante para realizar la taxonomía de grupos (MATTHEWS, 1974; NGUYENPHUNG \& CAMBEFORT, 1986a, 1986b, 1987; D'HOTMAN \& ZUNINO \& HALFFTER, 1988, 1990; SCHOLTZ, 1990; GÉNIER, 1996; KOHLMANN, 2000; GONZÁLEZ et al., 2009), es hasta la última década que se están trabajando estos caracteres en la taxonomía y filogenia de insectos.

En Scarabaeidae los caracteres internos del órgano copulador muestran una consistencia intraespecífica mayor que la que suele ocurrir en los caracteres morfológicos externos (ZUNINO \& MONTERESINO, 1990), mientras que estos últimos están fuertemente condicionados en su evolución por la presión selectiva del medio (GONZÁLEZ et al., 2009). La morfología de la genitalia interna del macho puede estar menos expuesta a selección por parte de condiciones ambientales, aunque puede estar influida directamente por selección sexual (ZUNINO, 1985; GONZÁLEZ et al., 2009). 
En insectos la genitalia masculina consiste básicamente de un órgano primario (edeago), cuya función es transferir el esperma a la hembra, y una serie de órganos secundarios (cerci), los cuales sirven para asir a la hembra durante la copulación (SCUDDER, 1959). Como caracteres taxonómicos diagnósticos de la genitalia masculina en Scarabaeinae se tienen en cuenta el edeago, el saco interno con sus escleritos y el segmento genital (MEDINA et al., 2013).

Por lo tanto, describir en detalle la variación morfológica del órgano genital masculino de las especies de la tribu Phanaeini presentes en Colombia incluyendo segmento genital, edeago y el saco interno fue el objetivo del presente trabajo, siendo una herramientas para la incorporación de nuevos datos taxonómicos al banco de información y en la conformación del inventario nacional de la biodiversidad.

\section{MATERIALES Y MÉTODOS}

Se revisaron 122 ejemplares de 55 especies, en 6 géneros y 25 especies de otros países como Costa Rica, Brasil, Perú, Bolivia y Venezuela depositados en las colecciones entomológicas del Instituto de investigaciones Alexander von Humboldt (IAvH), Villa de Leyva, Boyacá, Colombia; Colección Entomológica del Museo de Historia Natural "Luis Gonzalo Andrade”, Universidad Pedagógica y Tecnológica de Colombia, Tunja, Boyacá, Colombia (UPTC); Colección Escarabajos coprófagos de Colombia, (ECC) Bogotá; Colección Entomológica; Pontificia Universidad Javeriana Bogotá (MUJ).

Para el tratamiento taxonómico fueron consultadas las claves taxonómicas propuestas por EDMONDS (1972, 1994), VÍTOLO (2000), ARNAUD (2002), EDMONDS \& ZÍDEK (2004, 2010, 2012). Se realizó una descripción del órgano genital masculino de cada una de las especies disponibles, se revisó el edeago, saco interno y el segmento genital y se realizaron las ilustraciones del órgano genital de cada una de las especies estudiadas. La terminología que se utilizó para los escleritos del saco interno sigue la propuesta de MEDINA et al. (2003) y MEDINA et al. (2013).

El aislamiento de la genitalia masculina se realizó con los especímenes secos que se ablandaron mediante inmersión en agua caliente entre 15 minutos a media hora dependiendo del tamaño del espécimen. Bajo estereoscopio y con pinzas de disección finas se retiraron los órganos genitales del macho. El edeago, el saco interno y el segmento genital se sumergieron en $\mathrm{KOH}$ a una concentración del 5 y el 10\% entre 2 y 3 minutos, hasta poder ver con claridad los escleritos en el caso del saco interno, después se lavaron las estructuras mencionadas con agua y se guardaron en un frasco para genitales con glicerina (GONZÁLEZ et al., 2009).

\section{RESULTADOS Y DISCUSIÒN}

El órgano genital masculino de los Phanaeinos está conformado por varios elementos, el edeago en los Scarabaeinae es el término que se usa para referirse al órgano copulador del macho, este es una cápsula esclerotizada formada por una pieza basal, el lóbulo medio y los parámeros (Figura 1), que se ubican distalmente (SHARP \& MUIR, 1912; D'HOTMAN \& SCHOLTZ, 1990). El edeago ha tenido un valor taxonómico, en particular los parámeros su forma, ángulo y simetría. 
Dentro de la cápsula del edeago se encuentra el saco interno, este es una bolsa membranosa y alargada donde se distinguen diferentes zonas (basal, sub-medial, medial y apical) (MEDINA et al., 2013), en estas zonas se encuentran diferentes escleritos envueltos en una membrana gruesa. Para la tribu Phanaeini la zona submedial presenta un lóbulo y de acuerdo a la especie se presentan franjas donde la membrana está recubierta internamente de ráspulas de diversos tamaños y formas, en algunos casos se encuentran agrupaciones de setas que forman cepillos, en el extremo posterior del saco se encuentran dos láminas ligeramente esclerotizadas conocidas como temonas las cuales son dos placas aplanadas y de forma variable.

En cuanto a los escleritos del saco interno para las especies estudiadas de la tribu Phanaeini se encontraron cuatro, los cuales varían su forma, tamaño y en pocos casos la posición; en la zona medial y apical se encuentran los escleritos basal, placa y el esclerito elongado (Figura 1).

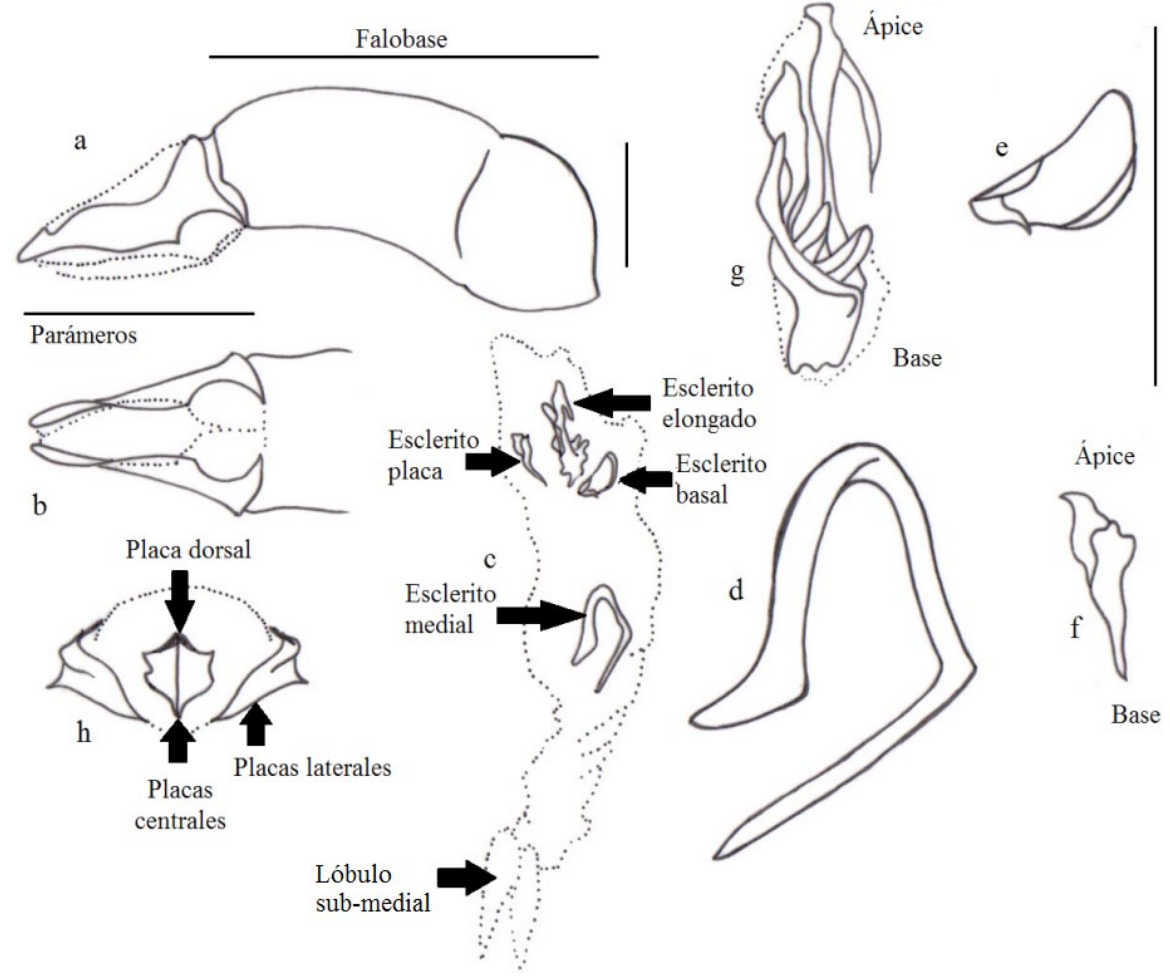

Escala: $1 \mathrm{~mm}$.

Figura 1. Oxysternon ebenium, morfología órgano genital masculino. a: Edeago, b: Parámeros, c: Saco interno, d: Esclerito medial, e: Esclerito basal, f: Esclerito placa, g: Esclerito elongado, h: Segmento genital.

Edeago. En la subfamilia Scarabaeinae el ángulo entre la falobase y los parámeros es de más de $110^{\circ}$ alcanzando los $180^{\circ}$ en grupos como Coprini, Onitini y Eucraniini (MEDINA et al., 2013). En el resto de las tribus la falobase forma un ángulo entre $90^{\circ}$ y $110^{\circ}$; en el caso de la tribu Phanaeini en la mayoría de las especies el ángulo 
que se forma es de $180^{\circ}$, solo en algunos casos como O. conspicillatum, O. lautum, C. spitzi, C. ignecinctus, P. kirbyi, S. menelas (Figuras 2-7) entre otros, el ángulo es de $45^{\circ}$; la mayoría de especies de Phanaeus tienen un ángulo de $170^{\circ}$ y solo en $C$. gamezi el ángulo entre los parámeros y la falobase es de $90^{\circ}$.

La tribu Phanaeini presenta parámeros simétricos, sin embargo, por ejemplo, los deltochilines de África y Australia poseen parámeros asimétricos aunque los del nuevo mundo presentan parámeros simétricos, algunos Deltochilum (GONZÁLEZ et al., 2009) y las especies del género Scybalocanthon (MOLANO \& MEDINA, 2010) también presentan los parámeros asimétricos.

La variación en la forma de los parámeros es amplia en los diferentes grupos de Scarabaeinae, para el caso de la tribu Phanaeini se observaron parámeros con formas triangulares y rectangulares, además algunas especies presentan variaciones como: proyecciones dorsales o ventrales como en $O$. festivum festivum, O. festivum viridanum, $S$. velutinus (Figuras 9-10); cuando las proyecciones son conspicuas y puntiagudas se define como un diente, este es el caso de $C$. conocephalus, $C$. corythus, $C$. telamón nevinsoni, $C$. dardanus, C. telamon, $C$. ignecinctus, $C$. parvulus, C. solisi y $C$. lancifer (Figura 13); en Canthon por ejemplo se definen cuatro tipos de formas de los parámeros: triangular, rectangular, forma de bota con un diente curvado en posición ventral y cara ventral sinuada (MEDINA et al., 2003).

Los parámeros pueden ser más cortos, iguales o más largos con respecto a la falobase; toda esta variación también se encontró en la tribu Phanaeini, en Oxyternon y Sulcophanaeus los parámeros con respecto a la falobase son más cortos, mientras que en algunas especies de Phanaeus y Coprophanaeus los parámeros son más largos o iguales que la falobase, como caracteres llamativos se encuentra una proyección membranosa en el ápice de los parámeros con ráspulas en forma de escamas en P. pyrois (Figura 12).

En general, los edeagos en Scarabaeinae son glabros pero en especies como Byrrhidium Harold, 1869 hay presencia de setas, en Phanaeini se observa la presencia de una sola seta en el ápice de los parámeros en S. auricollis auricollis. En otras especies de Scarabaeinae se presenta una fila de setas largas al lado de los parámeros (MEDINA et al., 2013). Otro carácter que presenta mucha variación en la forma, es el ápice de los parámeros que para Phanaeini se definen tres: redondeado, que en algunos caso como $P$. dejeani y $P$. palaeno se estrecha aunque la punta es redondeada, triangular o sub-triangular, y rectangular, la variación de estas formas la presenta $P$. kirbyi y $P$. beltianus en los que el ápice se aplana formando una especie de aleta.

Segmento genital. Estas placas varían en posición, orientación, forma y grado de esclerotización (MEDINA et al., 2013), varios autores han tomado características del segmento para la definición de especies y en filogenia (PHILIPS et al., 2002, 2004).

En las especies examinadas de la tribu Phanaeini, el segmento genital presenta bordes esclerotizados confluentes que pueden estar cubriendo parcial o totalmente las placas centrales, en general el ángulo externo de las placas laterales es de $90^{\circ} \mathrm{y}$ redondeado; haciendo que el segmento tenga una forma semicuadrada, sin embargo especies como $C$. corythus y $C$. lancifer presentan unas prolongaciones en el ángulo de los bordes laterales en forma de gancho; la variación en las placas laterales se 
presenta en el grado de espesor, por ejemplo en Arachnodes splendidus (Fairmaire, 1889) las placas laterales son delgadas y son mucho más gruesas en Gyronotus fimetarius Kolbe, 1894 (MEDINA et al., 2013).

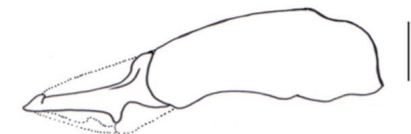

2

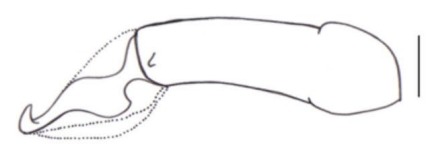

5

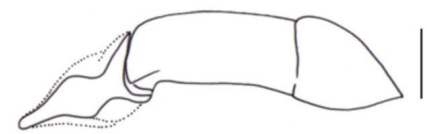

8

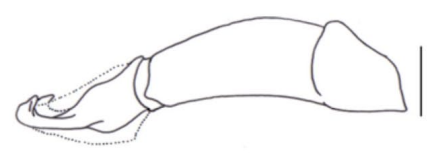

11

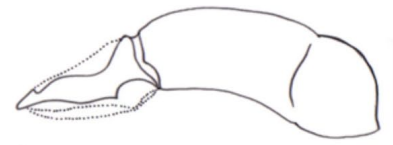

3
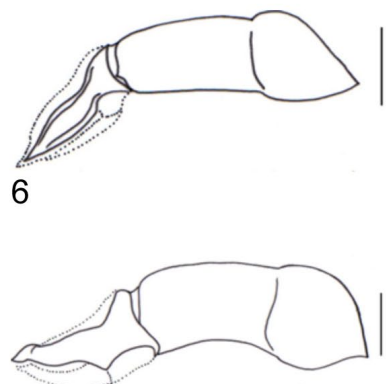

9

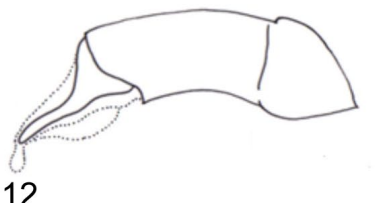

12

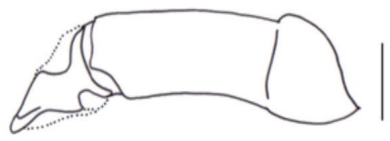

4
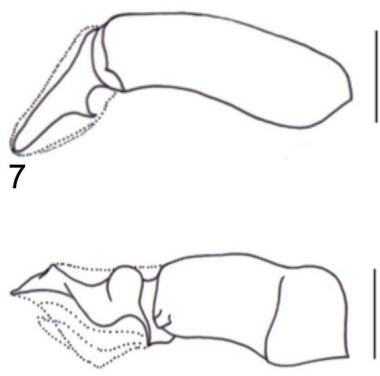

10

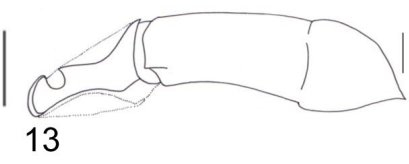

Figura 2-13. Edeago. 2: Oxysternon conspicillatum (Weber, 1801). 3: Oxysternon lautum (Macleay, 1819). 4: Coprophanaeus spitzi (Pessôa, 1934). 5: Coprophanaeus ignecinctus(Felshe, 1909). 6: Phanaeus kirbyi Vigors, 1825. 7: Sulcophanaeus menelas (Laporte, 1840). 8: Coprophanaeus gamezi Arnaud, 2002. 9: Oxysternon festivum festivum (Linné, 1767). 10: Sulcophanaeus velutinus (Murray, 1856). 11: Coprophanaeus corythus (Harold, 1863). 12: Phanaeuspyrois Bates, 1887. 13: Coprophanaeus lancifer (Linné, 1767).

Las placas centrales o placas mediales varían dentro de la subfamilia, la placa central puede variar en el grado de esclerotización, formar dos proyecciones o estar divididas en dos placas quitinizadas que se unen o también pueden estar completamente separadas, para las especies de la tribu se observó mucha variación de este carácter debido a que estas pueden tener forma semicircular, arriñonada, acorazonada, semicuadrada, triangular o una mezcla de estas formas, por ejemplo en los subgéneros de Deltochilum: Calhyboma, Hybomidium y Telhyboma las placas centrales se dividen en dos generando una bifurcación que forman a su vez unas áreas entre las dos placas (GONZÁLEZ et al., 2009), estas áreas entre las placas centrales en Phanaeini tienen formas triangulares, ovaladas, rectangulares, hasta forma de botella, y ello depende de la forma de las placas centrales.

Otra placa que se define adicional a las propuestas por MEDINA et al. (2013), es la placa dorsal pues en los segmentos genitales de las especies de la tribu Phanaeini se observa mucha variación en forma, en su disposición espacial con respecto a las 
placas centrales y además estas placas se internalizan recubriéndolas; en la tribu la placa dorsal puede adoptar formas trapezoidales, semicirculares o rectangulares.

Generalmente, el segmento genital no presenta estructuras accesorias como setas, espinas o demás piezas u ornamentaciones sobre las placas; para los subgéneros Calhyboma y Telhyboma de Deltochilum no se reportan estructuras accesorias en el segmento genital (GONZÁLEZ et al., 2009), igualmente en Scybalocanthon (MOLANO \& MEDINA, 2010) ni en Canthon (MEDINA et al., 2003) mientras tanto en la tribu Phanaeini algunas especies presentan setas en las placas centrales y laterales, se encuentran setas en las placas centrales en especies como $C$. telamon nevinsoni, $C$. pecki, C. telamon, C. morenoi, C. telamon telamon, $P$. kirbyi y $P$. haroldi y setas en las placas laterales se encuentran en $P$. kirbyi, $P$. pyrois, $P$. prasinus, $P$. meleagris minos, $P$. meleagris, $P$. palaeno, $C$. telamon nevinsoni, $C$. pecki, $C$. dardanus, $C$. telamon, C. morenoi, C. telamon telamon (Figuras 14-29). Ornamentaciones de las placas laterales se muestran en $S$. leander en donde se observa un cinturón quitinizado de bordes sinuados.

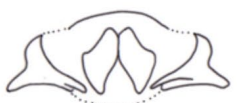

14

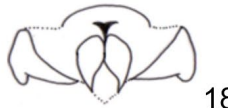

18

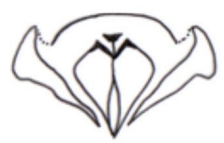

22

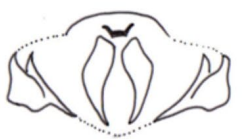

26

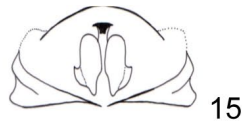

15

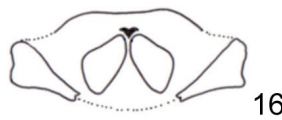

16
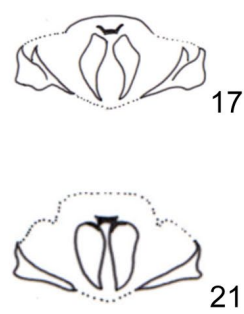

19

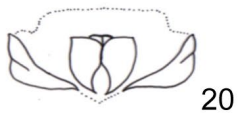

20
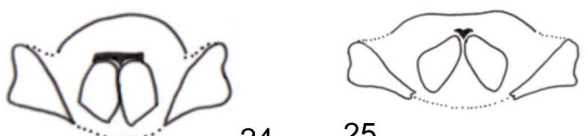

25

24

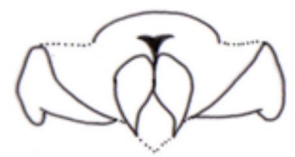

28

27

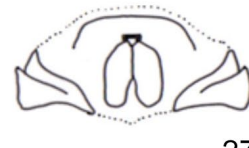

Figuras 14-29. Segmento genital. 14: Coprophanaeus corythus (Harold, 1863). 15: Coprophanaeus lancifer (Linné, 1767). 16: Coprophanaeus telamon nevinsoni Arnaud \& Gámez, 2002. 17: Coprophanaeus pecki Howden \& Young, 1981. 18: Coprophanaeus telamon (Erichson, 1847). 19: Coprophanaeus telamon telamon (Erichson). 20: Phanaeus haroldi Kirsch, 1870. 21: Phanaeus kirbyi Vigors, 1825. 22: Phanaeus pyrois Bates, 1887. 23: Phanaeus meleagris minos Erichson, 1847. 24: Phanaeus palaeno Blanchard 1846. 25: Coprophanaeus telamon nevinsoni Arnaud \& Gámez, 2002. 26: Coprophanaeus pecki Howden \& Young, 1981. 27: Coprophanaeus dardanus (McLeay, 1819). 28: Coprophanaeus telamon (Erichson, 1847).

Saco interno del edeago. De acuerdo a lo propuesto por MEDINA et al. (2013), el saco interno se ha dividido en cuatro regiones: basal, sub-medial, medial y apical, el área basal es la zona contigua a las temonas y generalmente desprovista de estructuras esclerotizadas. 
En el área sub-medial área contigua a la basal hay presencia de sacos de ráspulas, en el caso de la tribu Phanaeini estos sacos adoptan diversas formas, al igual que los tamaños y formas de las ráspulas definidas así por ZUNINO (1972) o cepillos del saco interno (MEDINA et al., 2003), por ejemplo en O. festivum, O. festivum viridanum, O. festivum festivum, O. conspicillatum, O. lautum y O. ebenium (Figuras 30-33) se observan setas largas, mientras que en $P$. haroldi (Figura 34) se presentan unas esclerotizaciones en forma de láminas alrededor del esclerito. En la gran mayoría de las especies de la tribu Phanaeini estas ráspulas cubren toda el área sub-medial al igual que en las especies Byrrhidium convexum Scholtz \& Howden, 1987, Ennearabdus lobocephalus Harold, 1868 (MEDINA et al., 2013).

Por otra parte, todas las especies de la tribu presentan en esa región un lóbulo corto cubierto de ráspulas en forma de escamas o espinas, este lóbulo ha sido observado también en especies de Scybalocanthon (MOLANO \& MEDINA, 2010) en otros Deltochilini como en Canthon bicolor (MEDINA et al., 2003), Canthon auricollis Redtenbacher, 1867 y las especies de Deltochilum siendo este lóbulo un poco más largo en este grupo (GONZÁLEZ et al., 2009).

En la región medial estructuras esclerotizadas pueden estar presentes o no, en las especies con presencia de escleritos el número de ellos es variable de uno a tres y la forma depende de cada especie; en la tribu Phanaeini en todas las especies se encuentra un solo esclerito con variación en la forma de acuerdo a la especie, mientras que en la tribu Deltochilini nunca se presenta este esclerito a excepción del género Deltochilum (MEDINA et al., 2013). En otros géneros como Onthophagus (BINAGHI et al., 1969), Dichotomius (LÓPEZ-GUERRERO, 2005) y en las tribus Eucraniini y Onitini siempre está presente el esclerito medial. La forma más común de este esclerito en la tribu Phanaeini es de media luna; para el género Sulcophanaeus este esclerito en forma de media luna presenta una proyección central y en algunos casos se ensancha en los extremos, en Oxysternon la forma en general es de $\underline{U}$ invertida al igual que en Diabroctis cadmus, en Coprophanaeus y Phanaeus la forma del esclerito varía desde formas de media luna, $\underline{\mathrm{U}}$ invertida, forma en espiral, en forma de $\mathrm{J}$ hasta forma de trípode.

En la zona apical se encuentra un grupo de escleritos; la mayoría de las especies tienen tres escleritos: el basal que está ubicado en la base de los otros dos escleritos, el esclerito elongado que generalmente es el más grande y con largas proyecciones, y el esclerito placa con diversidad de formas pero generalmente es ancho y plano. En las especies de la tribu Phanaeini en el ápice del saco interno se presenta una agrupación de ráspulas formando una $\underline{\mathrm{V}}$ invertida.

Esclerito basal. A este esclerito se le nombra esclerito circular por la forma que adoptaba en diferentes especies de Canthon y en la mayoría de los Deltochilini donde se desarrolló la terminología para las descripciones de este tipo de escleritos, por ejemplo se llama anillo a la parte circular de los escleritos y "mango" cuando la parte circular es acompañada por una extensión ampliada quitinosa (MEDINA et al., 2003), sin embargo revisando en la subfamilia Scarabaeinae se encontró variación en la forma de este esclerito; en las tribus Oniticelini, Onthophagini, Eucraniini, Coprini y Phanaeini el esclerito basal en estos grupos no tiene forma de anillo (MEDINA et al., 2013). En Phanaeini el esclerito basal está sobre un lóbulo corto, solo en $C$. lancifer el esclerito no se ubica sobre este, la disposición sobre el lóbulo hace que el esclerito sea cóncavo en la mayoría de los casos; las formas que puede 
adoptar el esclerito son variadas, la más común es redondeado con un mango en el extremo superior que varía de forma triangular, cuadrada a rectangular, en otros casos la forma es de media luna o semicircular, en Sulcophanaeus el esclerito está rodeado de ráspulas en forma de escamas mientras en Coprophanaeus los escleritos basales no están rodeados por ningún tipo de ráspulas.

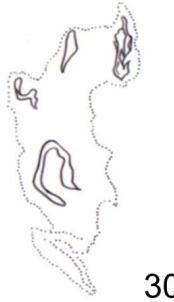

30

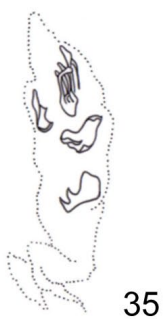

35

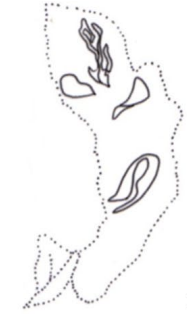

31

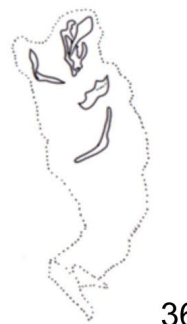

36
32

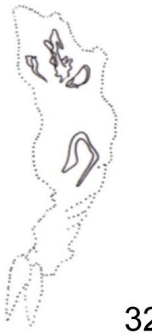

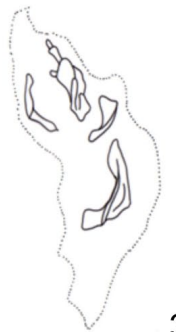

37

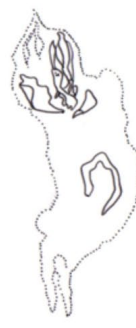

33

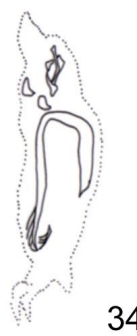

34

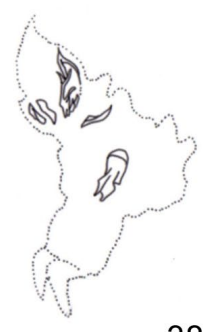

$38 \quad 39$

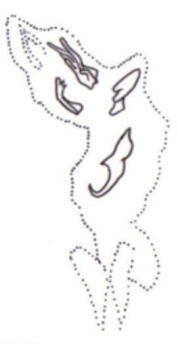

Figuras 30-39. Saco interno. 30: Oxysternon festivum viridanum Arnaud, 2002. 31: Oxysternon conspicillatum (Weber, 1801). 32: Oxysternon lautum (Macleay, 1819). 33: Oxysternon ebenium (Nevinson, 1890). 34: Phanaeus haroldi Kirsch, 1870. 35: Phanaeus prasinus Harold, 1868. 36: Coprophanaeus dardanus (McLeay, 1819). 37: Coprophanaeus bellicosus (Olivier, 1789). 38: Oxysternon silenus Laporte, 1840. 39: Sulcophanaeus miyashitai miyashitai Arnaud, 2002.

Esclerito placa. La presencia de este esclerito es constante en Scarabaeinae con un amplio rango de variación (MEDINA et al., 2013), esta estructura es plana con algunos pliegues o puntas de acuerdo a la especie, o en algunos casos son de estructuras simples como en Coptorhina excavata; dentro de los escleritos con estructura simple se encuentran los miembros de Phanaeini en el que las formas más comunes son de media luna, acorazonada o rectangular, además el esclerito habitualmente presenta una proyección en el borde dorsal central y en el caso de $S$. leander debajo del esclerito se encuentra un paquete de espinas largas conspicuas. Generalmente, el esclerito placa está protegido dorsalmente por una lámina de ráspulas en forma de escamas en este grupo. En otros casos, el esclerito placa está formado por placas alargadas superpuestas como es el caso de Onthophagini. Evaluando la forma que adopta el esclerito placa en algunos géneros se observan patrones, sin embargo en algunas especies aisladas taxonómica o a nivel biogeográfico, también muestran igual forma de esclerito placa como el caso de Gyronotus, Scybalophagus y en algunas especies de Scybalocanthon (MEDINA et al., 2013). 
Esclerito elongado. Este esclerito está presente en la mayoría de las tribus de Scarabaeinae, está formado por diferentes placas esclerotizadas superpuestas, que pueden estar fusionadas en una estructura sólida o en otros casos estas placas no se encuentran fusionadas. Para las especies de Phanaeini el esclerito elongado está compuesto por tres piezas que no se fusionan, las cuales varían de forma y tamaño de acuerdo a la especie, siendo unas más elaboradas o con más ornamentaciones; estas tres piezas se caracterizan por ser independientes, uniéndose en la base o en algunos casos hay proyecciones que se unen hacia los ápices. El esclerito hacia la base generalmente está recubierto por unas láminas de ráspulas con forma de escamas. Aunque no hay un patrón básico entre los diferentes géneros, en los géneros en ocasiones se observa un patrón en las estructuras que lo conforman. Para las especies de Canthon, MEDINA et al. (2003) encontraron una gran variación y no un patrón constante entre los escleritos observados, todo lo contrario ocurre entre las especies del género Epirinus.

\section{Algunas diferencias y similitudes en la genitalia masculina entre especies y subespecies de la tribu Phanaeini}

La genitalia masculina en el caso de las especies y subespecies de $O$. festivum, O. festivum viridanum y $O$. festivum festivum del género Oxysternon definidas por ARNAUD (2002) y EDMONDS \& ZÍDEK (2004), muestran diferencias entre las subespecies, por ejemplo en $O$. festivum la placa dorsal del segmento genital tiene forma de $\underline{U}$ invertida, la cual se internaliza entre las placas centrales recubriendo el borde interno de estas placas, en $O$. festivum viridanum la placa dorsal es de forma rectangular y no se internaliza en las placas centrales, y en $O$. festivum festivum la placa dorsal es rectangular con los extremos inferiores redondeados formando un arco y tampoco se internaliza en las placas centrales.

En $C$. pecki al igual que en $C$. morenoi el esclerito medial es muy reducido, casi desaparece, el esclerito tiene forma de media luna, en $C$. pecki además se observa un lóbulo adicional en el área del esclerito placa.

Mientras tanto, en las genitalias masculina de $S$. auricollis auricollis y $S$. auricollis joffrei hay diferencias en la presencia de una seta en el ápice de los parámeros y forma del esclerito placa, por caracteres morfológicos externos tanto EDMONDS (2000) como ARNAUD (2002) definen estas dos subespecies dentro del grupo auricollis.

La genitalia de las especies $C$. telamon, $C$. telamon telamon, $C$. corythus y $C$. telamon nevinsoni presenta diferencias a nivel del segmento genital, en $C$. corythus por ejemplo el ángulo externo de las placas laterales tiene un diente redondeado, en $C$. telamon se forma una protuberancia redondeada en el ángulo externo de estas placas, en las otras dos especies el ángulo externo es recto (Figura 28), en $C$. corythus la placa dorsal es poco esclerotizada, mientras que en las otras especies se forma una especie de $\underline{\mathrm{T}}$, adicionalmente se presentan setas cortas en las placas centrales del segmento genital de $C$. telamon nevinsoni, estas no se presentan en C. telamon y C. telamon telamon. El esclerito basal varía de forma en todas las especies mencionadas.

Con respecto a las especies $P$. meleagris y la subespecie $P$. meleagris minos la variación más evidente se presenta en la forma de las placas centrales, en $P$. 
meleagris es acorazonada mientras que P. meleagris minos es romboidal además de un ligero cambio de forma en el esclerito basal.

Por último, es interesante mostrar variaciones a nivel de ejemplares de diferentes zonas geográficas, por ejemplo se observó variación en la forma del esclerito medial entre individuos de $S$. faunus, los cuales eran de Colombia, el otro de Bolivia. Igualmente, se observó variación entre individuos del Perú y del Vaupés (Colombia) de la especies $O$. conspicillatum, en este caso la variación se presenta en la forma del esclerito placa y una ligera variación en el esclerito medial.

Tabla 1. Diferencias o similitudes de características morfológicas externas y la genitalia entre algunas especies y subespecies de la tribu Phanaeini

\begin{tabular}{lll}
\hline \multicolumn{1}{c}{ Subespecies } & \multicolumn{1}{c}{$\begin{array}{c}\text { Diferencias morfológicas } \\
\text { externas }\end{array}$} & \multicolumn{1}{c}{ Genitalia } \\
\hline \multicolumn{1}{c}{ Sulcophanaeus } \\
\hline S. auricollis auricollis & $\begin{array}{c}\text { EDMONDS (2000) define las } \\
\text { subespecies por coloración y tamaño. }\end{array}$ & $\begin{array}{l}\text { Presencia de seta en el ápice de } \\
\text { los parámeros en } S \text {. auricollis } \\
\text { ARNAUD (2002) las define por } \\
\text { auricollis, diferencias en la forma } \\
\text { S. auricollis joffrei }\end{array}$ \\
& poblaciones y coloración. & delito placa. \\
\hline
\end{tabular}

\begin{tabular}{|c|c|c|}
\hline S. noctis cupricollis & \multirow{2}{*}{$\begin{array}{l}\text { EDMONDS (2000) no reconoce a } \\
\text { S. noctis cupricollis como subespecie. } \\
\text { ARNAUD (2002) diferencia las } \\
\text { subespecies como S. noctis noctis y } \\
\text { S. noctis cupricollis por la coloración } \\
\text { del pronoto. }\end{array}$} & \multirow[b]{2}{*}{ No hay diferencias evidentes. } \\
\hline S. noctis & & \\
\hline S. miyashitai miyashitai & \multirow{2}{*}{$\begin{array}{l}\text { EDMONDS (2000) no define estas } \\
\text { subespecies. ARNAUD (2002) } \\
\text { las diferencia por poblaciones y } \\
\text { coloración. }\end{array}$} & \multirow[b]{2}{*}{ No hay diferencias evidentes. } \\
\hline S. miyashitai metallescens & & \\
\hline \multicolumn{3}{|c|}{ Oxysternon } \\
\hline O. festivum & \multirow{3}{*}{$\begin{array}{l}\text { ARNAUD (2002) diferencia } \\
\text { O. festivum viridanum por los } \\
\text { fémures completamente negros. } \\
\text { EDMONDS \& ZÍDEK (2004) } \\
\text { reconocen solo O. festivum festivum. }\end{array}$} & \multirow{3}{*}{$\begin{array}{l}\text { Diferencias en la forma de la } \\
\text { placa dorsal, en las tres especies, } \\
\text { varía la forma del esclerito placa. }\end{array}$} \\
\hline O. festivum festivum & & \\
\hline O. festivum viridanum & & \\
\hline
\end{tabular}

\section{Coprophanaeus}

\begin{tabular}{|c|c|}
\hline C. telamon & \\
\hline C. telamon telamon & reconocen a $C$. telamony $C$. telamon \\
\hline C. telamon nevinsoni & telamon, y C. telamon nevinsoni pasó \\
\hline & $\begin{array}{l}\text { a C. corythus. ARNAUD (2002) por } \\
\text { su parte reconoce las dos subespecies } \\
\text { y propone } C \text {. telamon corythus. }\end{array}$ \\
\hline C. corythus & $\begin{array}{l}\text { Las divisiones las hace a partir de } \\
\text { ensanchamiento de la carina cefálica } \\
\text { y torácica. }\end{array}$ \\
\hline
\end{tabular}

C. telamon, C. telamon telamon y C. telamon nevinsoni presentan setas en las placas laterales y centrales del segmento genital, varía la forma del esclerito placa en todas las subespecies. Las placas laterales de C. corythus tienen un ángulo redondeado. 


\begin{tabular}{|c|c|c|}
\hline Subespecies & $\begin{array}{c}\text { Diferencias morfológicas } \\
\text { externas }\end{array}$ & Genitalia \\
\hline \multicolumn{3}{|c|}{ Phanaeus } \\
\hline P. meleagris & EDMONDS \& ZÍDEK (2012) & \\
\hline P. meleagris minos & $\begin{array}{l}\text { no reconocen a } P \text {. meleagris minos. } \\
\text { ARNAUD (2002) propone esta } \\
\text { subespecie basado en la presencia de } \\
\text { punturaciones en el disco pronotal } \\
\text { de los machos. }\end{array}$ & $\begin{array}{l}\text { Se observan diferencias en la } \\
\text { forma de las placas centrales } \\
\text { y diferencias en la forma del } \\
\text { esclerito placa. }\end{array}$ \\
\hline
\end{tabular}

\section{CONCLUSIONES}

Pocos estudios se han realizado sobre la genitalia masculina en las especies de la tribu Phanaeini. A pesar del carácter descriptivo del presente trabajo, se han mostrado las variaciones en la morfología de los componentes de la genitalia masculina, edeago, saco interno, segmento genital y los escleritos, generando nuevos caracteres morfológicos internos útiles para una mejor comprensión de las relaciones filogenéticas tanto de la tribu como de la subfamilia Scarabaienae.

Los caracteres aquí explorados brindan las herramientas en la búsqueda y selección de caracteres apropiados para los análisis cladísticos junto con caracteres de la morfología externa, estudios moleculares, así como aspectos de la biología de las especies. Sin embargo, se recomienda tener en cuenta caracteres aún no explorados como forma, ubicación y agrupación de las ráspulas.

\section{BIBLIOGRAFÍA}

ANICHTCHENKO, A.V., 2004.- Utilización de la estructura de saco interno del edeago en carábidos (Insecta, Coleóptera, Carabidae) para la taxonomía. Disponible en: http://paganel-net.xm.com/utilizacion.htm ARNAUD, P., 2002.- Les Coléoptères du Monde 28. Phanaeini. Hillside Books, Canterbury, U.K.

BINAGHI, G., DELLACASA, G. \& POGGI, R., 1969.- Nuovi Caratteri diagnostici per la determinazione degli Onthophagus del gruppo ovatus (L.) e geonemia controllata delle specie Italiane del grupo. Memorie de lla Società Entomologica Italiana, Genova, 48, 29-46.

CAMBEFORT, Y., 1991.- From saprophagy to coprophagy: 22-35 (in) HANSKI, I. \& CAMBEFORT, Y. (eds.) Dung Beetle Ecology. Princeton University Press, Princeton, $\mathrm{Nj}$.

CÓRDOBA-AGUILAR, A., 2000.- Evolución y diversidad de la morfología de los genitales masculinos en insectos. Folia Entomológica Mexicana, 110: 95-111.

D'HOTMAN, D. \& SCHOLTZ, C.H., 1990.- Phylogenetic significance of the structure of the external male genitalia in the Scarabaeoidea. Entomology Memoir, Department of Agricultural Development, 77: 1-51.

EDMONDS, W.D., 1972.- Comparative skeletal morphology, systematics and evolution of the phanaeine dung beetles (Coleoptera: Scarabaeidae). The University of Kansas Science Bulletin, 49: 731-874.

, 1994.- Revision of Phanaeus Macleay, a new world genus of Scarabaeine dung beetles (Coleoptera: Scarabaeidae: Scarabaeinae). Natural History Museum of Los Angeles County, Contributions in Science, 443: 1-105.

, 2000.- Revision of the neotropical dung beetle genus Sulcophanaeus (Scarabaeidae: Scarabaeinae). Folia Heyrovskyana, 6:1-60.

EDMONDS, W.D. \& ZÍDEK, J., 2004.- Revision of the neotropical dung beetle genus Oxysternon (Scarabaeidae: Scarabaeinae: Phanaeini). Folia Heyrovskyana, 11: 1-58.

2010.- A taxonomic review of the Neotropical genus Coprophanaeus Olsoufieff, 1924 (Coleoptera: Scarabaeidae, Scarabaeinae). Insecta Mundi, 129: 1-111.

, 2012.- Taxonomy of Phanaeus revisited: Revised keys to and comments on species of the New World dung beetle genus Phanaeus MacLeay, 1819 (Coleoptera: Scarabaeidae: Scarabaeinae: Phanaeini). Insecta Mundi, Paper 784.

GÉNIER, F., 1996.- A revision of the Neotropical genus Ontherus. Memoirs of the Entomological Society of 
Canada, 170: 1-169.

GONZÁLEZ, F.A., MOLANO, F. \& MEDINA, C.A., 2009.- Los subgéneros Calhyboma Kolbe 1893, Hybomidium Shipp 1897 y Telhyboma Kolbe 1893 de Deltochilum (Coleoptera: Scarabaeidae: Scarabaeinae) en Colombia. Revista Colombiana de Entomología, 35: 253-274.

HALFFTER, G., 1991.- Historical and ecological factors determining the geographical distribution of Beetles (Coleoptera: Scarabaeidae: Scarabaeinae). Folia Entomológica Mexicana, 82: 195-238.

HALFFTER, G. \& FAVILA, M.E., 1993.- The Scarabaeinae (Insecta: Coleoptera) an animal group for analysing, inventorying and monitoring biodiversity in tropical rainforest and modified landscapes. Biology International, 27: 15-21.

HALFFTER, G. \& MATTHEWS, E.G., 1966.- The natural history of dung beetles of the subfamily Scarabaeinae. Folia Entomológica Mexicana, 12-14: 1-312.

KOHLMANN, B., 2000.- New species and distribution records of Mesoamerican Ateuchus. Revista de Biología Tropical, 48 (1): 235-246.

LÓPEZ-GUERRERO, Y., 1999.- Morphological comparison of the male genitalia of Eurysternus Dalman and Sisyphus Latreille (Coleoptera, Scarabaeidar, Scarabaeinae). The Coleopterists Bulletin, 53 (3): 201-207. , 2005.- Los Dichotomius (Coleoptera: Scarabaeidae, Dichotomini) de la fauna de México. Boletín Sociedad Entomológica Aragonesa, 36: 125-209.

MATTHEWS, E.G., 1974.- A revision of the Scarabaeine dung beetles of Australia II. Tribe Scarabaeini. Australian Journal of Zoology, Supplementary Series, Melbourne, 24: 1-211.

MEDINA, C.A., LOPERA-TORO, A., VÍTOLO A. \& GILL, B., 2001.- Escarabajos coprófagos (Coleoptera: Scarabaeidae: Scarabaeinae) de Colombia. Biota Colombiana, 2 (2): 131-144.

MEDINA, C.A., MOLANO, F. \& SCHOLTZ, C.H., 2013.- Morphology and terminology of dung beetles (Coleoptera: Scarabaeidae: Scarabaeinae) male genital. Zootaxa, 3626 (4): 455-476.

MEDINA, C.A., SCHOLTZ, C.H. \& GILL, B.D., 2003.- Morphological variation and systematics of Canthon and related genera of new world Canthonini dung beetles. Deutsche Entomologische Zeitschrift, Berlin, 50 (1): 23-68

MOLANO, F. \& MEDINA, C.A., 2010.- Especie nueva de Scybalocanthon (Coleoptera: Scarabaeinae: Canthonini) y descripción de la variación del órgano genital masculino. Revista Mexicana de Biodiversidad, 81: 689-699.

NGUYEN-PHUNG, T \& CAMBEFORT, Y., 1986a.- Révision des espèces afro-tropicales du genre Copris. I. Le groupe integer Reiche. Nouvelle Revue d'Entomologie, Paris (NS), 3 (4): 401-416.

1986b.- Révision des espèces afrotropicales du genre Copris Müller, 1764. III. Le groupe inhalatus Quedenfeldt (Coleoptera, Scarabaeidae). Revue Française d'Entomologie, (N.S.), 8(3): 100-106.

1987.- Révision des espèces afrotropicales du genre Copris Müller, 1764. II. Le groupe jacchus (Fabricius) (Coleoptera Scarabaeidae s. str.). Annales de la Société entomologique de France, 23(1): 67-75.

NORIEGA, J.A, CAMERO, E., ARIAS-BURITICÁ, J.A., PARDO-LOCARNO. L.C., MONTES, J.M., ACEVEDO, A.A. et al., 2015.- Grado de cobertura del muestreo de escarabajos coprófagos (Coleoptera: Scarabaeidae: Scarabaeinae) en Colombia. Revista Biología Tropical, 63 (1): 97-125.

PESSÔA, S.B., 1934.- Contribuição para o conhecimento das espécies brasileiras do gênero Phanaeus (Col. Scarabaeidae). Annaes da Faculdade da Medicina de São Paulo, 10: 279-314.

PHILIPS, T.K., SCHOLTZ, C.H. \& OCAMPO, F.C., 2002.- A phylogenetic analysis of the Eucraniini (Coleoptera: Scarabaeidae: Scarabaeinae). Insects Systematic and Evolution, 33: 241-252.

PHILIPS, T.K., EDMONDS, W.D. \& SCHOLTZ, C.H. 2004. A phylogenetic analysis of the New World tribe Phanaeini (Coleoptera: Scarabaeidae: Scarabaeinae): hypotheses on relationships and origins. Insect Systematics and Evolution, 35:43-63.

PRICE, D.L., 2009.- Phylogeny and biogeography of the dung beetle genus Phanaeus (Coleoptera: Scarabaeidae). Systematic Entomology, 34: 137-150.

SCUDDER, G.G.E., 1959.- The female genitalia of the Heteroptera: Morphology and bearing on classification. Trans. R. Entomol. Soc. London, 111 (14): 405-467.

SHARP, D. \& MUIR, F.A.G., 1912.- The comparative anatomy of the male genital tube in Coleoptera. Transactions of Entomological Society of London, 3: 477-642.

SPECTOR, S., 2006.- Scarabaeine dung beetles (Coleoptera: Scarabaeidae: Scarabaeinae): an invertebrate focal taxon for biodiversity research and conservation. Coleopterists Bulletin, 60: 71-83.

VÍTOLO, A., 2000.- Clave para la identificación de los géneros y especies de Phanaeinas (Coleoptera: Scarabaeidae: Coprinae: Phanaeini) de Colombia. Revista de la Academia Colombiana de Ciencias Exactas, Físicas y Naturales, 24 (93): 591-603.

ZUNINO, M., 1985.- Las relaciones taxonómicas de los Phanaeina (Coleoptera, Scarabaeinae) y sus implicaciones biogeográficas. Folia Entomológica Mexicana, 64: 101-115.

1972.- Revisione delle specie paleartiche del genere Onthophagus Latr. (Coleoptera Scarabaeoidea). I. Il. sottogenere Euonthophagus Balth. Bollettino del Museo di Zoologia dell'Università di Torino, 1: 1-28.

ZUNINO, M. \& HALFFTER, G., 1988.- Análisis taxonómico ecológico y biogeográfico de un grupo americano de Onthophagus. Museo Regionale di Scienze Naturale Torino Monografía, 9: 1-211.

ZUNINO, M. \& MONTERESINO, E., 1990.- Ideas preliminares sobre la evolución de los aparatos copuladores en Rutelinae (Coleoptera, Scarabaeoidea) y la filogenia del grupo. Revista de la Sociedad Entomológica Argentina, 48 (89): 3-13. 